\title{
Evaluation of visible light communication channel delay profiles for automotive applications
}

\author{
SeokJu Lee ${ }^{1}$, Jae Kyun Kwon', Sung-Yoon Jung ${ }^{*}$ and Young-Hoon Kwon ${ }^{2}$
}

\begin{abstract}
In this article, we present channel delay profiles based on simulated data regarding the practical conditions for the use of visible light communication (VLC) in automotive applications such as Intelligent Transportation Systems (ITS). Practical vehicular LED headlamp and street lamp that consider the lighting regulation for transportation are used to design the ITS scenarios based on VLC. We modeled two usage scenarios, crossroad and metropolitan street, using the CATIA V5 tool. Measurements for the VLC channel delay profile evaluation were then gathered by using a ray-tracing scheme employing commercial LightTools software under the vehicle-to-vehicle and vehicle-to-infrastructure (V2I) communication links. From the obtained channel impulse responses from both scenarios, we derived the VLC channel delay profiles. From them, we found that the common property of the delay profile was composed of dominant multiple line of sight (LOS) links and a less number of non-LOS delay taps. However, the channel delay profile for the V2I link and metropolitan scenario show more dispersive channel characteristics due to the reflection and diffusion of the visible light.
\end{abstract}

\section{Introduction}

Next-generation LED lighting has more advantages than existing fluorescent and incandescent lighting in terms of its long life expectancy, high tolerance to humidity, low-power consumption, and minimal heat generation. Recently, there have been many attempts to combine LED with IT technology. Among them, visible light communication (VLC), the convergence of illumination and communication, has been developed [1-6]. In general, VLC uses intensity modulation with direct detection scheme, which uses the intensity of the light to transmit data. Human eyes perceive only the average intensity when the light modulates faster than the maximum flickering time period, defined as $5 \mathrm{~ms}$ [3]. Therefore, both acceptable lighting and communication can be implemented simultaneously. The corresponding VLC standardization has recently been published by the IEEE standard association [3]. VLC has many applications in indoor lighting used for home and office environments and in outdoor lighting for vehicular environment. The VLC applications are summarized in the VLC standard group [4].

\footnotetext{
* Correspondence: syjung@ynu.ac.kr

${ }^{1}$ Department of Electronic Engineering, Yeungnam University, 214-1

Dae-dong, Gyeongsan-si, Gyeongsangbuk-do 712-749, Republic of Korea Full list of author information is available at the end of the article
}

Among these applications, the adaptation of VLC for Intelligent Transportation Systems (ITS) is of high interest to various VLC research groups [5-12]. In order to obtain intelligence in transportation, ITSs have utilized the advantages of the Information and Communication Technologies (ICT), providing several different technological systems that help users. Among the possible ICTs, ITSs are now considering the use of VLC, focusing on two aspects. First of all, it is possible to repurpose the transportation lighting infrastructure for communication. Recently, the transportation lighting infrastructure such as street lamps, traffic lights, automotive lamps, etc., is changing to LEDs. Therefore, in the case of an ITS based on VLC, it will be possible to make use of the conventional automotive and traffic LEDs. Consequently, the cost incurred in constructing the ITS infrastructure will be reduced. Second, the electro magnetic compatibility problem, which is a very serious problem in ITSs based on RF signals, will be minimized since visible light and the conventional RF signals occupy different parts of the electromagnetic spectrum.

To adopt VLC in an ITS, the first requirement is to know the VLC channel characteristics in the ITS scenario. In the IEEE VLC standard [4], the technical consideration document outlines the "Types of Channels" for VLC in regards to automotive applications. However, it does not discuss 


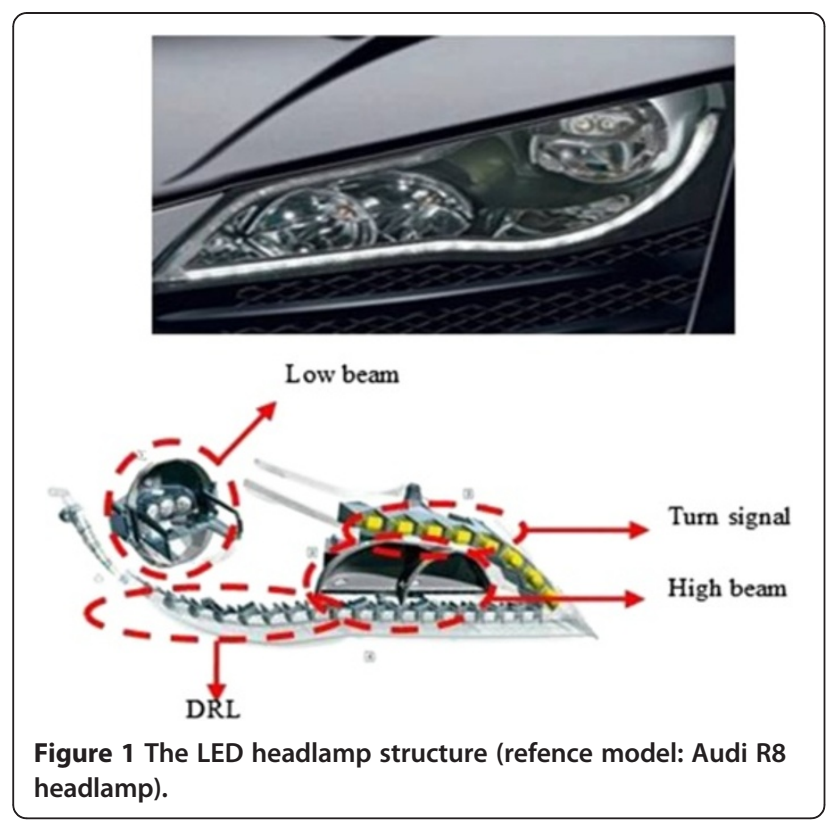

any channel parameter details that describe the reference channel model. The related research papers use either a mathematical channel model that utilizes the simplified automotive environments [7-9], or use their own developed channel modeling simulators, which still contain many great simplifications and assumptions [10-12].

In this article, we present a VLC channel delay profiles based on simulated data regarding the practical ITS usage scenario. In order to describe the practical VLC channel more precisely, we considered the lighting regulation for transportation, such as the light distribution

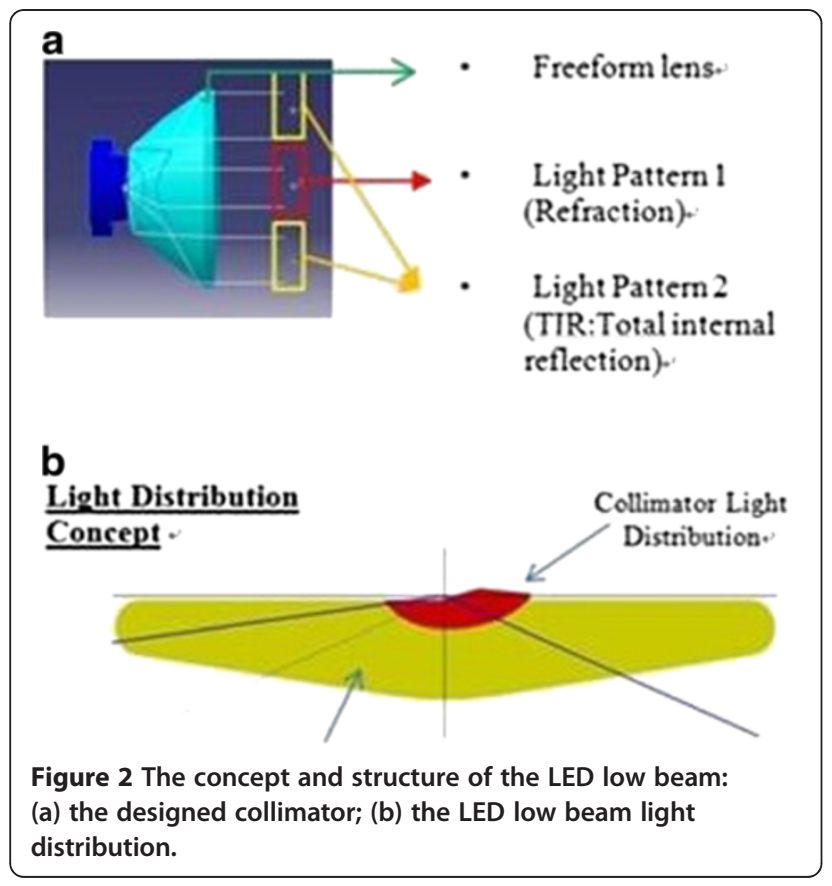

for vehicular LED headlamps and street lamps and other optical properties when designing the ITS scenario. For the simulations, two urban usage scenarios, crossroad and metropolitan street, were modeled using the CATIA V5 tool [13]. Measurements for VLC channel delay profile evaluation were gathered using a Ray-Tracing scheme employing LightTools [14] software, a widely accepted commercial optical engineering tool, in vehicle-to-vehicle (V2V) and vehicle-to-infrastructure (V2I) cases. These tools are efficient when determining optical channel models, since they provide visual understanding and enable the practical modeling of simulation environments. We used the Lambertian reflection property for the reflectance parameter of each object for interference effect and reflectance in order to provide a more realistic scene. It is known that the reflection pattern of most surfaces can correctly be approximated using the Lambert or the Phong models. If there are strong specular components in the reflection pattern of the surfaces, the Phong model approximates those patterns well unlike that of the Lambert model $[15,16]$. However, the surface conditions in urban areas are irregular and reflect optical signals without favoring any particular direction. Therefore, because the reflection patterns are diffuse, they can correctly be approximated using the Lambertian reflection model. Finally, the channel delay profiles were evaluated from the measurements. From the derived channel delay profiles, we can observe that the

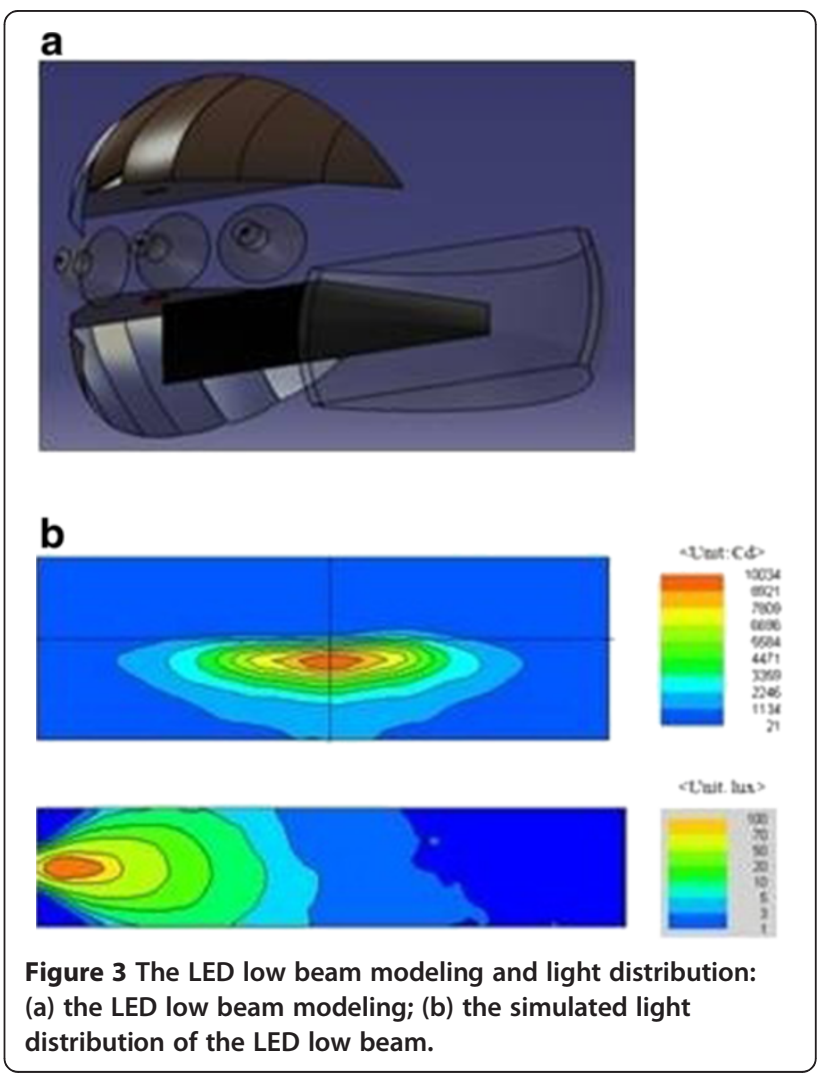



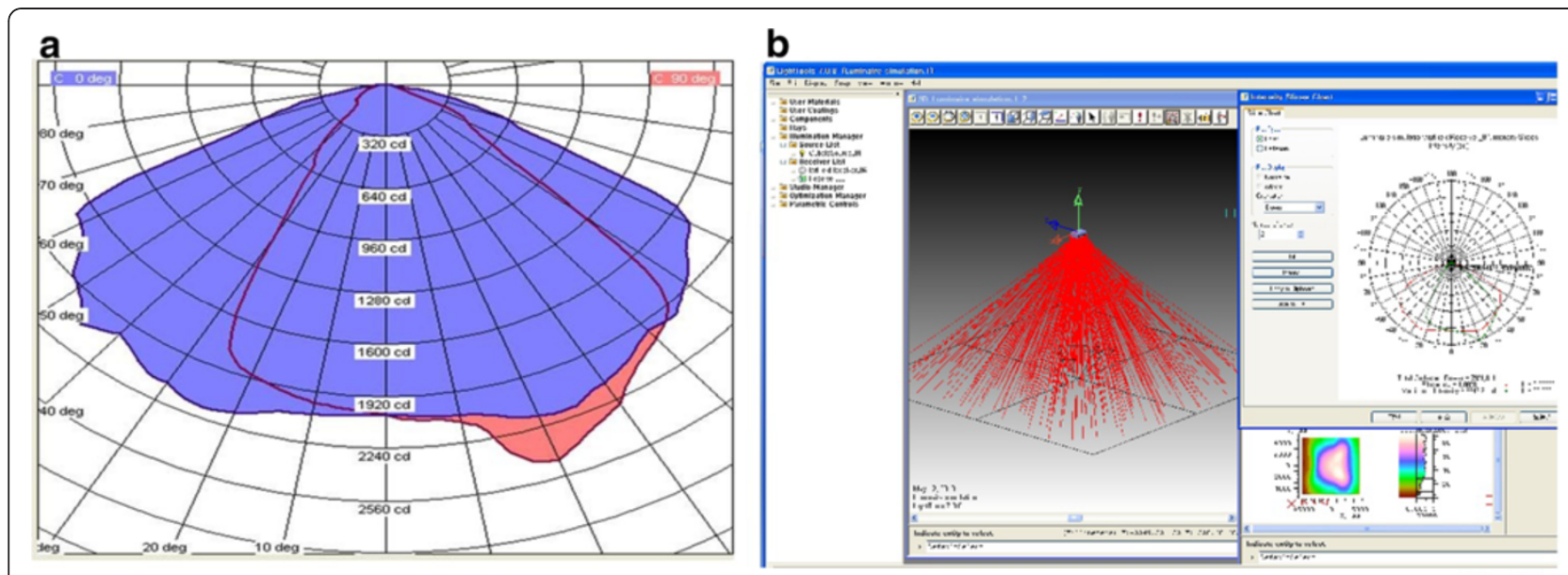

Figure 4 The LED street lamp light distribution: (a) the light distribution data; (b) the simulated light distribution.

common property of the delay profile was composed of dominant multiple line of sight (LOS) links and a less number of non-LOS (NLOS) delay taps. However, the delay profile for the V2I link and metropolitan scenario shows greater dispersive channel characteristics due to the reflection and diffusion of the visible lights.

\section{The VLC channel environment modeling}

\subsection{The ITS VLC light source modeling}

We model two practical LED light sources for VLC in the ITS, an automotive LED headlamp and an LED street lamp.

An LED headlamp is composed of a high beam, low beam, turn signal lamp, position lamp, and a daytime running lamp, as shown in Figure 1. In order to achieve a more precise modeling, we use a practical lamp model for the light sources. By using the modeling data of the practical LED headlamp, we design the low beam of an LED headlamp. Because the LED source intensity is less than a conventional halogen or HID, many LEDs are needed for an equivalent lamp performance. For this reason, LED headlamps consist of multiple sources and optical components. Furthermore, the sizes of the sources and optical components are smaller than those found in conventional lamps. In the case of our reference LED headlamp and the low beam module have three collimating optics using lenses and two spread optics using a reflector. Five LEDs are used with a projection lens and
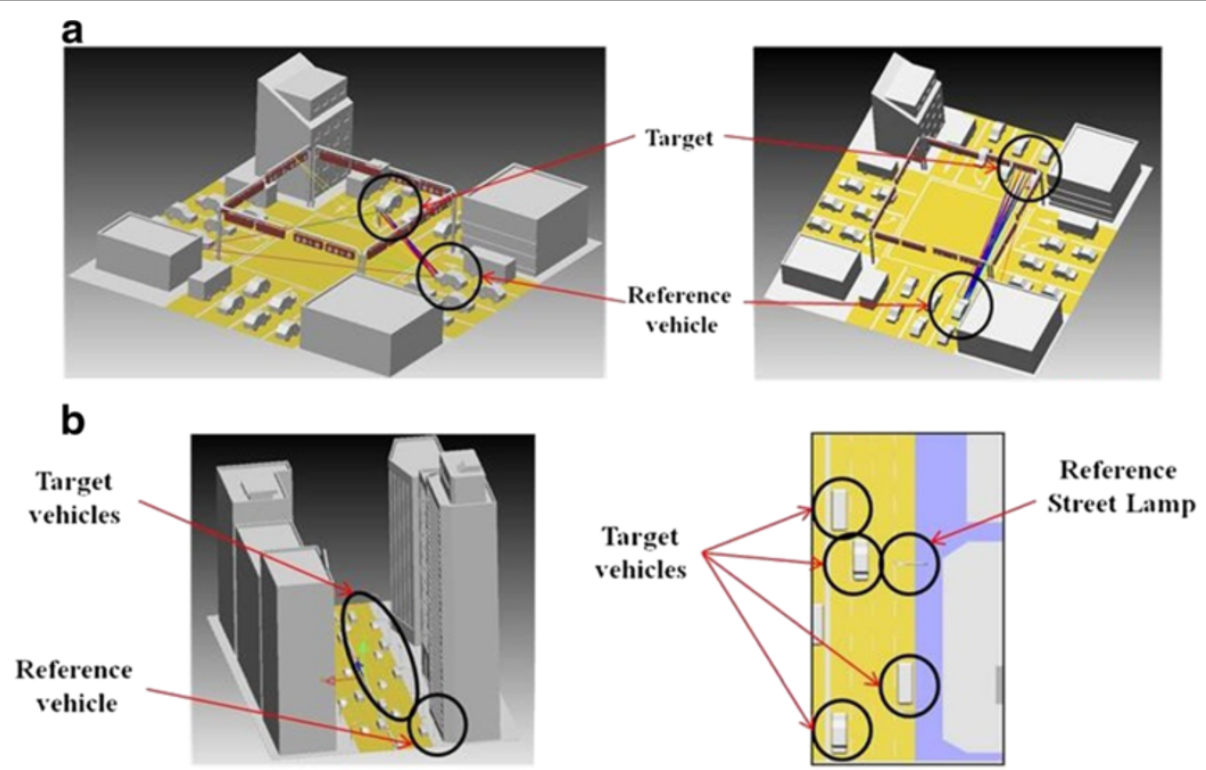

Figure 5 The VLC ITS usage scenarios: (a) the crossroad scenario; (b) the metropolitan street scenario. 

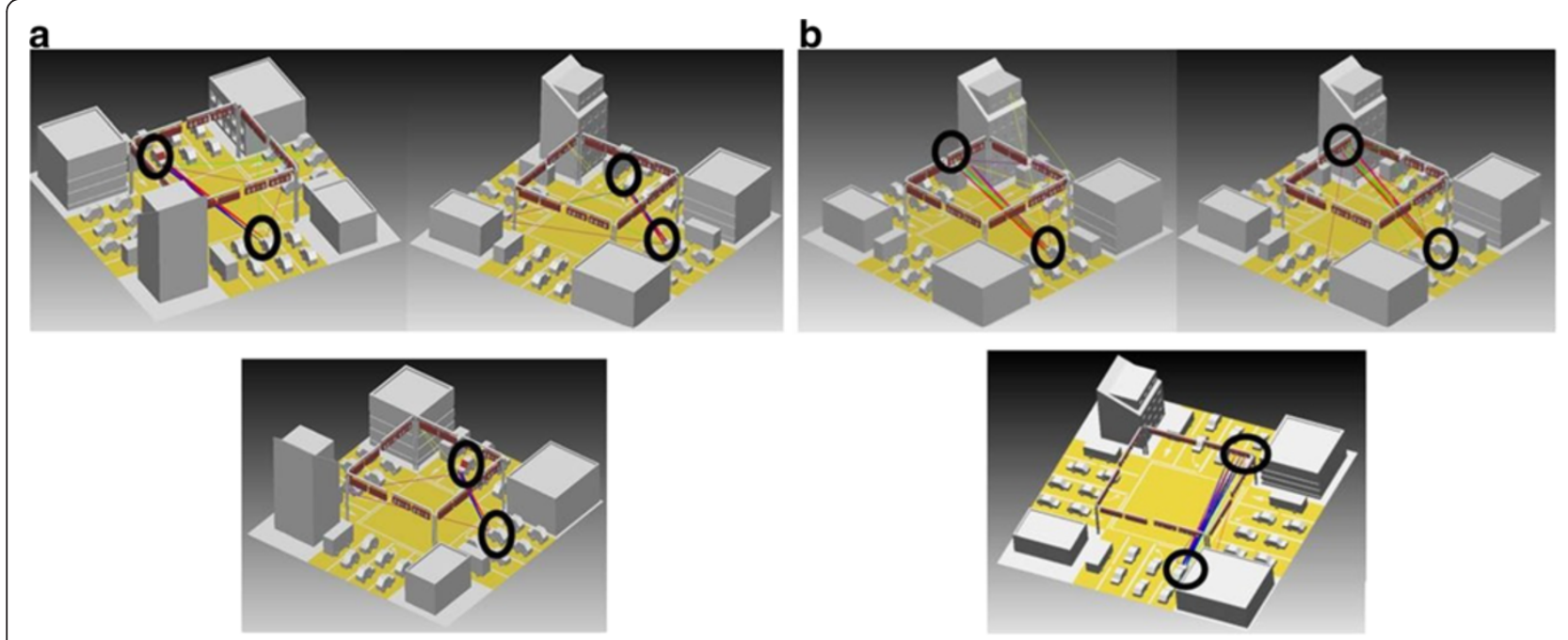

Figure 6 The VLC scenes for the V2V and V2I communication links in crossroad scenario: (a) the three scenes for the V2V communication link; (b) the three scenes for the V2I communication link.

cutoff shield to shape the light distribution of the LED low beam. Figure 2 shows the collimator design and the light distribution of the low beam. Finally, we design low beam module using CATIA V5 tool. After setting the three collimating and two spread optics, we run the optic simulation. The corresponding light distribution is optimized using SPEOS CATIA CAA based on EC regulations, as shown in Figure 3.

The other practical light source was the street lamp. We imported the light distribution of the LED street lamp from the Dialux library instead of modeling the real shape. Figure 4 shows the light distribution data and the simulated light distribution of the LED street lamp.

\subsection{The ITS VLC usage scenario modeling}

We assume that ITSs using VLC will initially be deployed in urban areas. This is because a great deal of LED lighting infrastructure such as LED street lamps, LED traffic lights, and LED lamps in vehicles have started to be deployed in urban areas. As a consequence, we choose two VLC usage scenarios for the ITS, as shown in Figure 5.

The first is a crossroad scenario composed of vehicles running in four directions, buildings, roads/fields, and traffic lights. Vehicles waiting for the traffic signals in crossroad can exchange information with the traffic lights and other vehicles, such as traffic information,

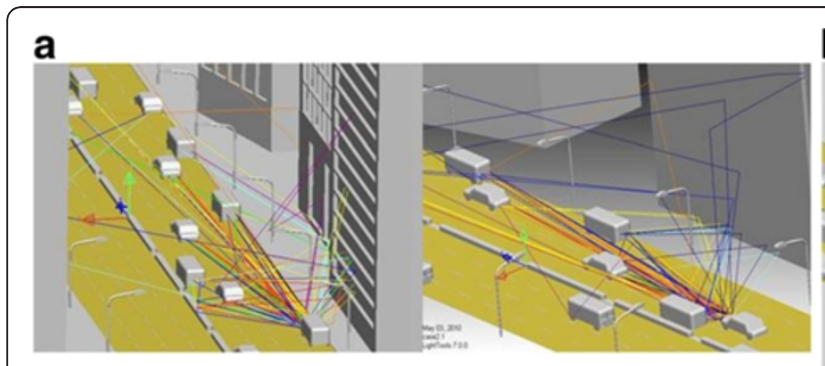

\section{b}
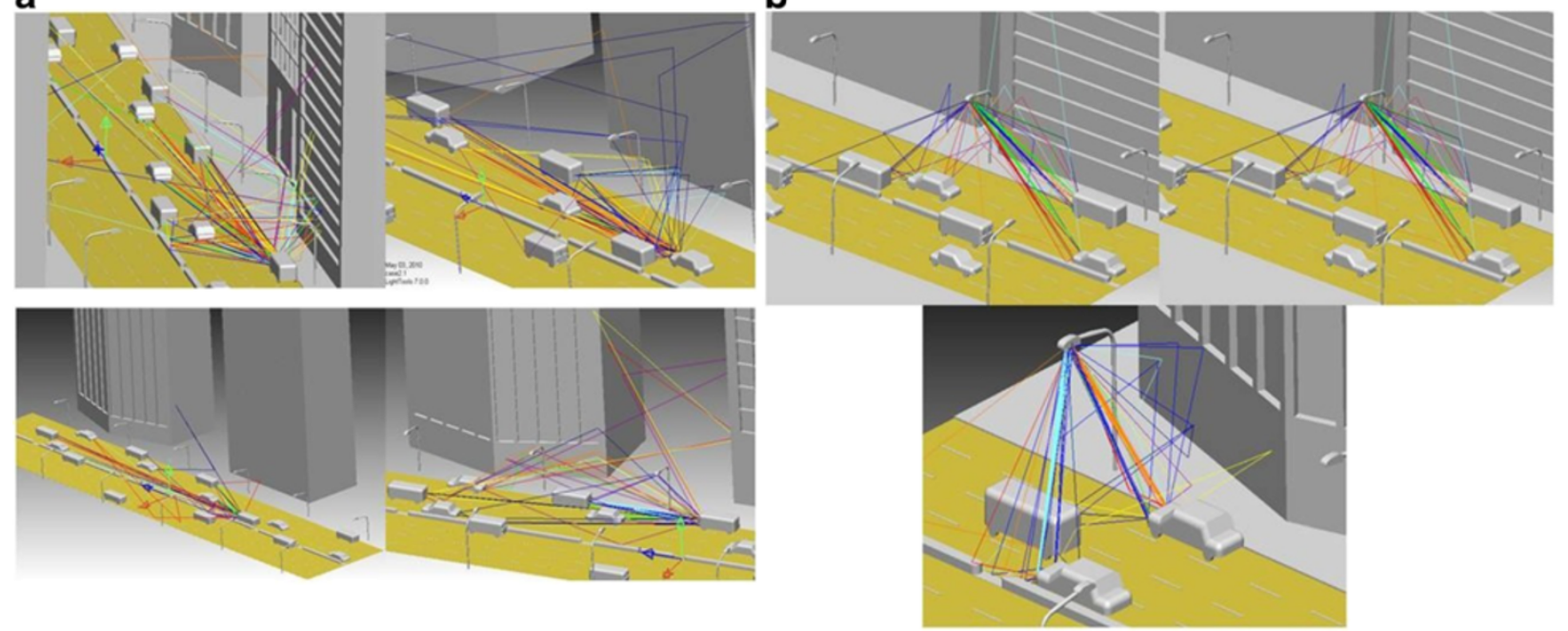

Figure 7 The VLC scenes for the V2V and V2I communication link in metropolitan street scenario: (a) the four scenes for the V2V communication link; (b) the three scenes for the V2I communication link. 
vehicle conditions, etc. The second scenario is a metropolitan street, which has a longer street area, higher buildings, vehicles, and street lamps. The second scenario is the more important ITS service scenario, since many vehicles in urban streets will benefit from several types of traffic information in order to enhance the traffic flow efficiency. Other important service scenarios, i.e., a high-way scene, may easily be applied based on the metropolitan street scenario. Note that the two proposed scenarios consider both V2V and V2I communication links.

In the first scenario, we design a scene using CATIA V5 tools. It is then imported to LightTools for the optic simulations. After this initial process, the optic simulation process is performed as follows: First, the reference cars as a light source and target, traffic lights as detectors, and other objects as interference environments are set. Next, the parameters used to reflect the optical

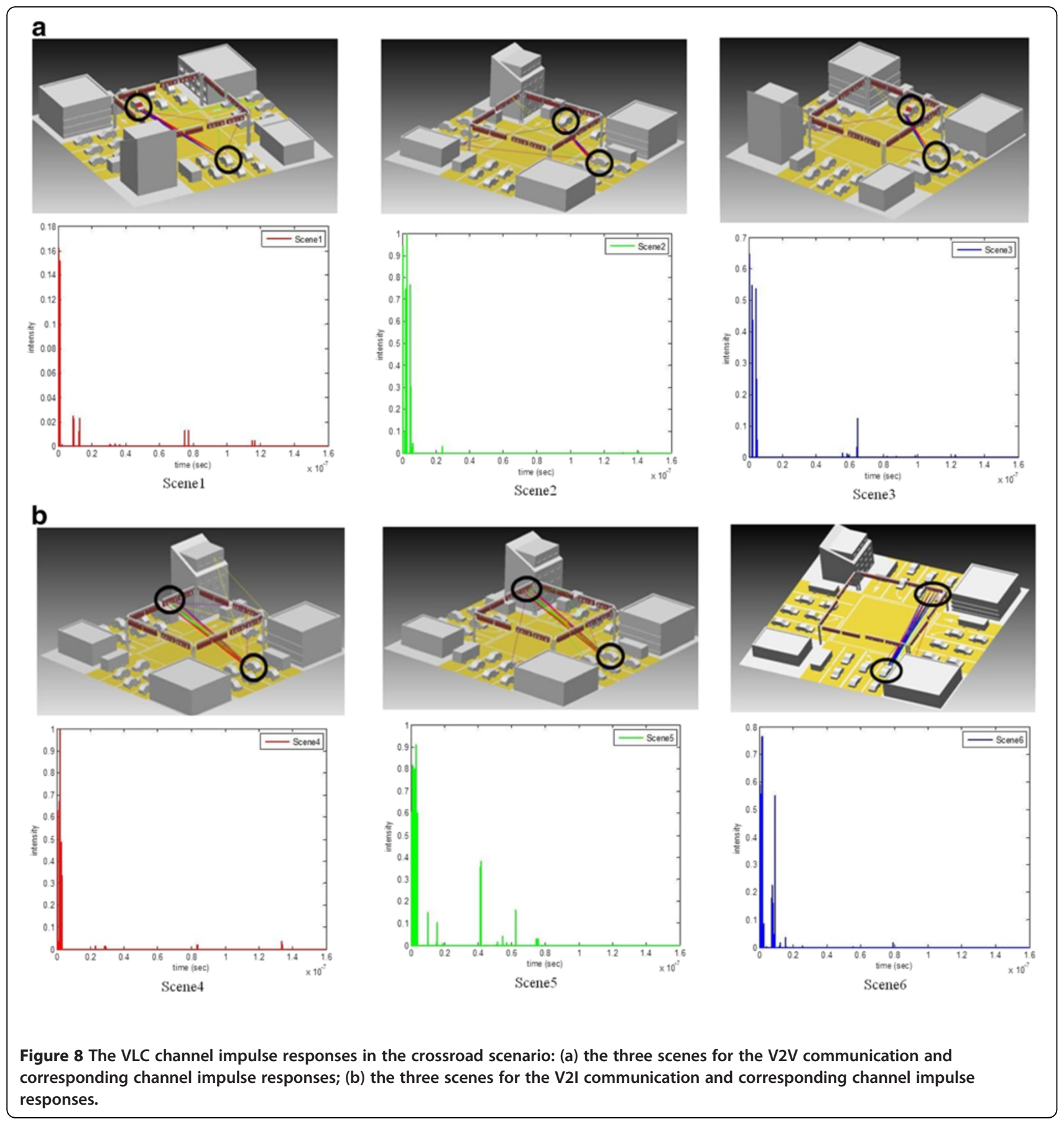


characteristics of the objects that constitute the scene are determined. We apply the Lambertian reflection property to the buildings, poles, cars, and road lines in order to provide a more realistic scene. We set the reflectance to $40 \%$ for the buildings, $10 \%$ for the poles, $20 \%$ for the cars, and $30 \%$ for the road lines. Then remaining reflectance percentages are set as absorptions. In the case of roads, they are set to fully be absorbed. For the light sources, we import the light distribution of the practical headlamp as discussed in Section 2.1. In order to evaluate the channel delay profile, we use a total of six scenes (three for the V2V and three for the V2I communication link), as shown in Figure 6.

In the second scenario, the initial process is the same with as that found in the first scenario. In this case, we also deal with V2V and V2I communication links. For the V2V link modeling, we set the reference vehicle first. We then determine the target vehicles in the field-ofview (FOV) range of the reference vehicle. In case of the V2I link modeling, we set four target vehicles under the a
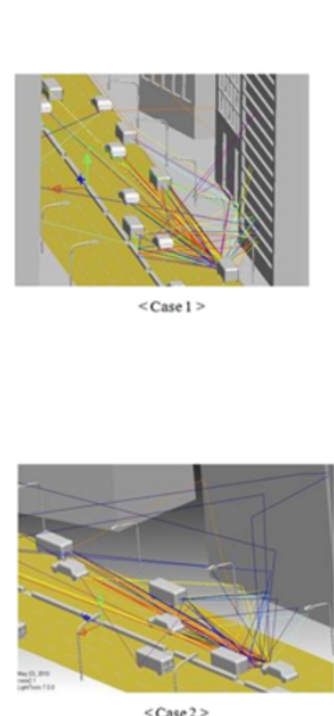

$<$ Case $2>$

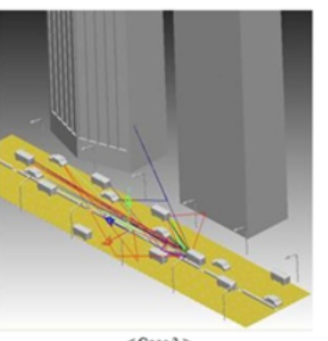

$\langle$ Case $3>$

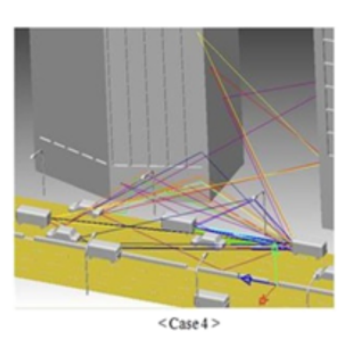

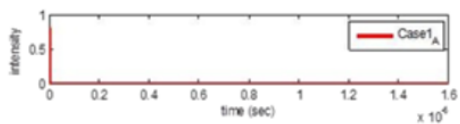
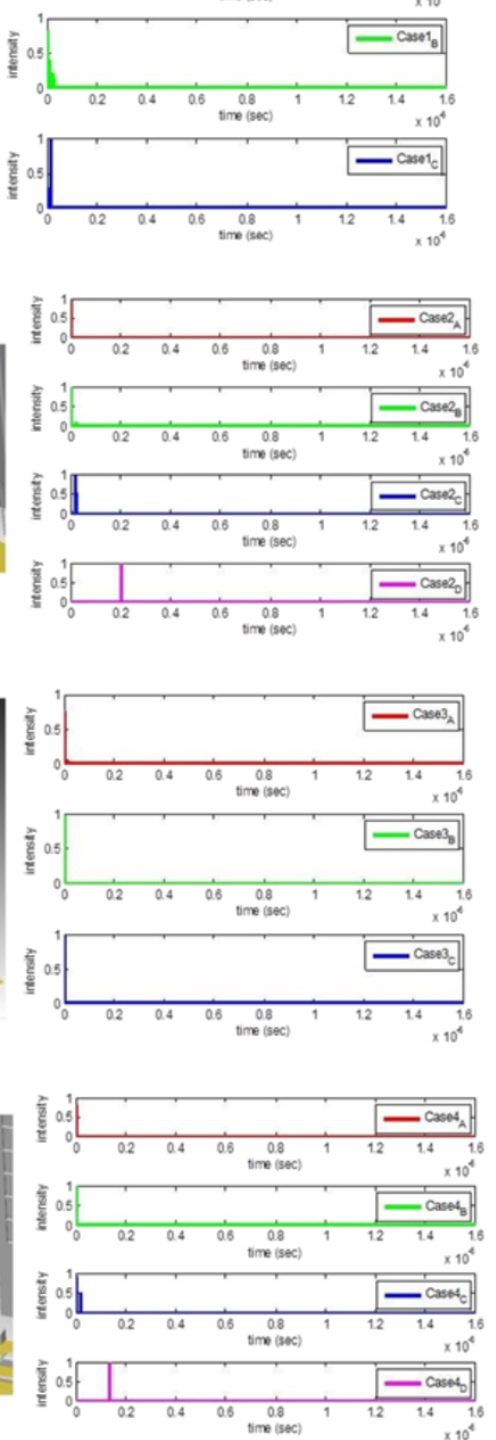

b
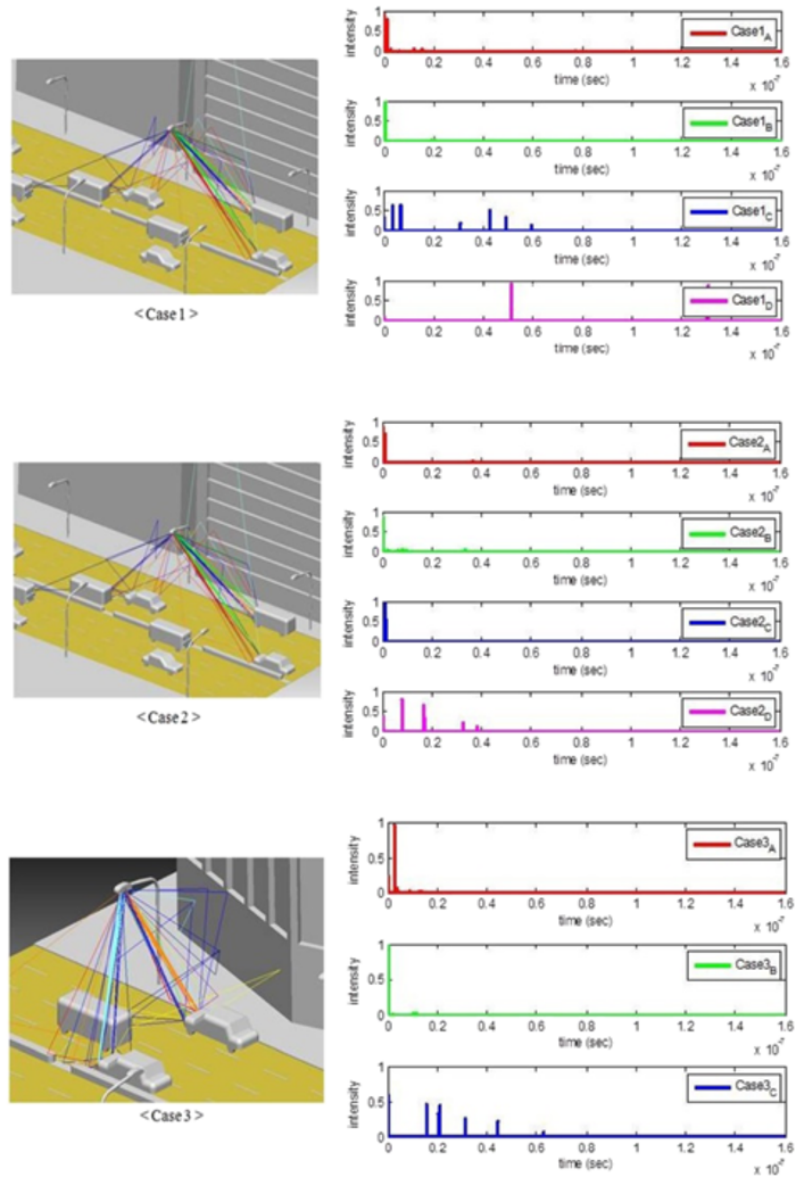

Figure 9 The VLC channel impulse responses in the metropolitan street scenario: (a) the four scenes for the V2V communication and corresponding channel impulses; (b) the three scenes for the V2V communication and corresponding channel impulses. 


\begin{tabular}{|c|c|c|c|c|}
\hline \multirow[t]{2}{*}{ Tap } & \multicolumn{2}{|c|}{ Crossroad-V2V } & \multicolumn{2}{|c|}{ Crossroad-V2I } \\
\hline & $\begin{array}{l}\text { Relative } \\
\text { delay (ns) }\end{array}$ & $\begin{array}{l}\text { Average } \\
\text { intensity (dB) }\end{array}$ & $\begin{array}{l}\text { Relative } \\
\text { delay (ns) }\end{array}$ & $\begin{array}{l}\text { Average } \\
\text { intensity (dB) }\end{array}$ \\
\hline 1 & 5 & -1.5362 & 5 & -2.2538 \\
\hline 2 & 10 & -24.2537 & 10 & -21.1482 \\
\hline 3 & 15 & -38.0701 & 15 & -33.8224 \\
\hline 4 & - & - & 20 & -45.8281 \\
\hline
\end{tabular}

reference street lamp after setting it. In the street lamp case, we use the practical street lamp light distribution described in Section 2.1. When evaluating the channel delay profile, we use a total of seven scenes (four for the $\mathrm{V} 2 \mathrm{~V}$ and three for the V2I communication link), as shown in Figure 7.

\section{The VLC channel simulation and delay profile evaluation}

\subsection{The VLC channel simulation results}

We consider two given scenarios (crossroad and metropolitan) for the VLC channel simulations. We use the CATIA V5 and LightTools software tools for evaluating the simulated VLC channel impulse responses. Using a backward optic ray tracing scheme, we determine the received optic power and the traveling length of each direct and indirect optic rays. These are then converted to channel impulse responses. All of the channel impulse responses are normalized to have the unit energy, and the propagation delay time is removed to represent the delay spread of each channel. Figure 8 shows the six scenes (three for V2V and three for V2I) in the crossroad scenario and the corresponding channel impulse responses. In the case of the V2V communication link, there are dominant multiple LOS paths with a negligible amount of NLOS delay taps. Therefore, this channel type can be considered to be a type of a multiple LOS channel. In the case of the V2I communication link, there are several LOS ingredients and a small number of NLOS delay taps. Therefore, it can be considered to be a type of as mixed LOS and NLOS channel.

Figure 9 shows the seven scenes (four for the V2V and three for the V2I) from the metropolitan scenario and

Table 2 The VLC channel delay profile of the metropolitan street scenario

\begin{tabular}{llllll}
\hline Tap & \multicolumn{2}{l}{ Metropolitan-V2V } & & \multicolumn{2}{l}{ Metropolitan-V2I } \\
\cline { 2 - 3 } \cline { 5 - 6 } & $\begin{array}{l}\text { Relative } \\
\text { delay }(\mathbf{n s})\end{array}$ & $\begin{array}{l}\text { Average } \\
\text { intensity }(\mathbf{d B})\end{array}$ & & $\begin{array}{l}\text { Relative } \\
\text { delay }(\mathbf{n s})\end{array}$ & $\begin{array}{l}\text { Average } \\
\text { intensity (dB) }\end{array}$ \\
\hline 1 & 5 & -7.5192 & 5 & -1.2254 \\
2 & 10 & -6.7048 & 10 & -31.0548 \\
3 & 30 & -100.2527 & 25 & -27.8063 \\
\hline
\end{tabular}

the corresponding channel impulse responses. As shown in the figure, there are several sub-cases for each scene. This is because we consider all of the target vehicles in the FOV range of the reference vehicle and streetlamp. Therefore, each sub-case describes the channel impulse response between the reference vehicle (or street lamp) and each target vehicle in the range of the FOV. In the V2V communication link, there are multiple LOS and NLOS delay taps with a short delay spread. Similarly, the V2I channel impulse response is composed of multiple LOS and NLOS delay taps with relatively more dispersive delay spread than the $\mathrm{V} 2 \mathrm{~V}$ link in some cases. Therefore, we determine that the V2V and V2I channel type for the metropolitan scenario to be mixed LOS and NLOS channels. Compared to the crossroad case, the metropolitan scenario shows more dispersive VLC channel conditions. In addition, the V2I link compared to the V2V link has more NLOS delay taps and a relatively more dispersive delay spread.

\subsection{The VLC channel delay profile evaluation}

From the simulated channel impulse responses from both scenarios, we evaluate the VLC channel delay profiles. When deriving the channel delay profile, we set the sampling frequency of the channel to $200 \mathrm{MHz}$. Tables 1 and 2 show the delay profiles of the VLC channels in the V2V and V2I cases from both scenarios.

From the tables, we can observe that the delay spreads from the metropolitan street scenario are more dispersive than those from the crossroad scenario because a great amount of diffused optical rays due to the interference objects produce NLOS delay taps with more dispersive delays. In addition, the V2I cases in both scenarios show more dispersive delay spreads than the $\mathrm{V} 2 \mathrm{~V}$ cases. This is because there are strong LOS optical ray components caused by the LED headlamp in the V2V cases.

\section{Conclusion}

Channel models are the essential baselines when we evaluate the basic performance of VLC systems through simulation. For the use of VLC in automotive ITS applications, we derived the VLC channel delay profiles based on the simulated data using LightTools and CATIA V5 software. These are efficient tools used to obtain optical channel models providing visual understanding and reflect practical modeling of simulation environments. For a more detailed modeling of the two urban usage scenarios (the crossroad and metropolitan scenarios), the shape and optical characteristics of the practical light sources (the LED headlamp and street light) and the infrastructure (buildings, roads/fields, vehicles) were used. From the derived channel delay profiles, we can observe that the common property of the delay profile was composed of 
dominant multiple LOS links and a less number of NLOS delay taps. However, the delay profile from the V2I link and metropolitan scenario had more dispersive channel characteristics due to the reflection and the diffusion of the visible light. Regarding future work, it will be required to measure a real VLC channel under the crossroad and metropolitan street scenarios and compare them to our simulated results. Because it requires a great deal of effort to measure a real channel, it will be meaningful to verify the validity of our channel evaluation schemes to confirm that it will provide a very useful tool for obtaining VLC channels in several operation scenarios.

\section{Competing interests}

The authors declare that they have no competing interests.

\section{Acknowledgments}

This study was supported by the Yeungnam University Research Grant.

\section{Author details}

Department of Electronic Engineering, Yeungnam University, 214-1

Dae-dong, Gyeongsan-si, Gyeongsangbuk-do 712-749, Republic of Korea.

${ }^{2}$ LED-IT Fusion Technology Research Center, 214-1 Dae-dong, Gyeongsan-si,

Gyeongsangbuk-do 712-749, Republic of Korea.

Received: 27 March 2012 Accepted: 6 November 2012

Published: 27 December 2012

\section{References}

1. T Komine, M Nakagawa, Fundamental analysis for visible-light communication system using LED lights. IEEE Trans. Consum. Electron. 50(1), 100-107 (2004)

2. G Ntogari, T Kamalakis, JW Walewski, T Sphicopoulos, Combining illumination dimming based on pulse-width modulation with visible-light communications based on discrete multitone. J. IEEE/OSA 3(1), 56-65 (2011)

3. IEEE Std 802.15.7, IEEE Standard for Local and metropolitan area networksPart 15.7, Short-Range Wireless Optical Communication Using Visible Light (IEEE, Piscataway, NJ, 2011)

4. R Richard D, L Sang-Kyu, IEEE 802.15.7 Visible Light Communication, Modulation Schemes and Dimming Support. IEEE Commun. Mag 50(1), 72-82 (2011)

5. M Rahaim, A Miravakili, T Borogovac, TDC Little, V Joyner, Demonstration of a software Defined Visible Light Communication System, in the 17th Annual International Conference on Mobile Computing and Networking, Mobicom2011 (2011)

6. T Komine, JH Lee, S Haruyama, M Nakagawa, Adaptive equalization system for Visible Light Wireless Communication utilizing multiple white LED lighting equipment. IEEE Trans. Wireless Commun 8, 2892-2900 (2009)

7. M Wada, T Yendo, T Fujiii, M Tanimoto, Road-to-vehicle communication using LED traffic light, in Intelligent Vehicles Symposium, 2005

8. T Nagura, T Yamazato, M Katayama, T Yendo, T Fujii, H Okada, Improved decoding methods of visible light communication system for ITS using LED array and high-speed camera, in Vehicular Technology Conference (VTC 2010Spring, 2010)

9. HCN Premachandra, T Yendo, MP Tehrani, T Yamazato, H Okada, T Fujii, M Tanimoto, High-speed-camera image processing based LED traffic light detection for road-to-vehicle visible light communication, in Proceedings of IEEE Intelligent Vehicles Symposium, 2010, pp. 793-798

10. IE Lee, ML Sim, FWL Kung, Performance enhancement of outdoor visiblelight communication system using selective combining receiver. IET Optoelectron 3(1), 30-39 (2009)

11. N Kumar, D Terra, N Lourenco, LN Alves, RL Aguiar, Visible light communication for intelligent transportation in road safety applications, in Proceedings of the IEEE International conference on Wireless Communications and Mobile Computing, 2011, pp. 1513-1518

12. IE Lee, ML Sim, FWL Kung, A dual-receiving visible light communication system under time-variant non-clear sky channel for intelligent transportation system, in Proceedings of IEEE European Conference on Network and Optical Communications, 2011, pp. 153-156

13. NA Davidoff, A graphic model of the human hand using CATIA. International Journal of Industrial Ergonomics 12, 255-264 (1993)

14. MJ Hayford, SR David, Characterization of illumination systems using LightTools. Proc. of SPIE 3130, 209 (1997)

15. CR Lomba, RT Valadas, AM de Oliveira Duarte, Efficient simulation of the impulse response of the indoor wireless optical channel. Int. J. Commun. Syst 13(7-8), 537-549 (2000)

16. CR Lomba, RT Valadas, AM de Oliveira Duarte, Experimental characterisation and modelling of the reflection of infrared signal on indoor surfaces. IET Proc. Optoelectron 145(3), 191-197 (1998)

doi:10.1186/1687-1499-2012-370

Cite this article as: Lee et al.: Evaluation of visible light communication channel delay profiles for automotive applications. EURASIP Journal on Wireless Communications and Networking 2012 2012:370.

\section{Submit your manuscript to a SpringerOpen ${ }^{\odot}$ journal and benefit from:}

- Convenient online submission

- Rigorous peer review

- Immediate publication on acceptance

- Open access: articles freely available online

- High visibility within the field

- Retaining the copyright to your article

Submit your next manuscript at $>$ springeropen.com 